\title{
Lack of Effect of Lovastatin Therapy on the Parameters of Whole-Body Cholesterol Metabolism
}

\author{
Ira J. Goldberg, * Stephen Holleran," Rajasekhar Ramakrishnan," Marian Adams, * \\ Robert H. Palmer," Ralph B. Dell, ${ }^{\ddagger}$ and DeWitt S. Goodman* \\ Departments of Medicine* and Pediatrics ${ }^{\ddagger}$ and the Arteriosclerosis Research Center, \\ College of Physicians and Surgeons, Columbia University, New York 10032
}

\begin{abstract}
The effects of lovastatin therapy on the parameters of body cholesterol metabolism were explored in nine hypercholesterolemic patients. Long-term cholesterol turnover studies were performed before therapy, and were repeated after 15 mo of lovastatin therapy $(\mathbf{4 0} \mathrm{mg} / \mathrm{d}$ ) while continuing on therapy. The major question addressed was whether a reduction in plasma cholesterol level with lovastatin would be associated with a reduction in the whole-body production rate of cholesterol or with the sizes of exchangeable body cholesterol pools as determined by the three-pool model of cholesterol turnover. The mean plasma cholesterol level decreased 19.4\% (from 294 to $237 \mathrm{mg} / \mathrm{dl}$ ), and low-density lipoprotein cholesterol decreased 23.8\% (from 210 to $159 \mathrm{mg} / \mathrm{dl}$ ) with lovastatin therapy. Changes in high-density lipoprotein cholesterol level were not significant. The cholesterol production rate did not change significantly with therapy $(1.09 \pm 0.10[$ mean \pm S.D.] vs. $1.17 \pm 0.09$ $\mathrm{g} / \mathrm{d})$. By comparison, colestipol and niacin treatment in three other subjects more than doubled the cholesterol production rate (1.14 \pm 0.28 vs. $2.42 \pm 0.34 \mathrm{~g} / \mathrm{d})$. Thus, hydroxymethylglutaryl-coenzyme A (HMG-CoA) reductase inhibition by lovastatin at the therapeutic dose used here did not change the steady-state rate of whole-body cholesterol synthesis. Despite the changes in plasma cholesterol levels, no significant changes were seen in the values of $M_{1}$, of $M_{3}$ or of $M_{\text {tot }}$, the sizes of the pools of rapidly, of slowly, and of total body exchangeable cholesterol. Conclusion: lovastatin therapy to lower plasma cholesterol does not lead to corresponding reductions in body cholesterol pools or to a reduction in the rate of whole-body cholesterol synthesis. In the new steady state that exists during long-term lovastatin therapy, along with increased expression of the genes for HMG-CoA reductase and the LDL receptor, the body compensates for the effects of the drug so that cholesterol production rate and tissue pool sizes are not changed from pretreatment values. (J. Clin. Invest. 1990. 86:801-808.) Key words: cholesterol turnover $\bullet$ hydroxymethylglutaryl-coenzyme A - hypercholesteremia - kinetic analysis • lovastatin $\bullet$ low density lipoprotein receptor
\end{abstract}

\section{Introduction}

Lovastatin is a member of a new class of potent plasma cholesterol-lowering agents which are competitive inhibitors of the

Address reprint requests to Dr. Goodman, Department of Medicine, Columbia University, 630 West 168th Street, New York, NY 10032.

Received for publication 16 January 1990 and in revised form 16 April 1990.

J. Clin. Invest.

(c) The American Society for Clinical Investigation, Inc.

0021-9738/90/09/0801/08 $\$ 2.00$

Volume 86, September 1990, 801-808 enzyme 3-hydroxy-3-methylglutaryl coenzyme A (HMGCoA $)^{1}$ reductase (1). This enzyme catalyzes the rate limiting step in cholesterol biosynthesis, the conversion of HMG-CoA to mevalonic acid. Inhibition of HMG-CoA reductase would be expected to lower cellular concentrations of cholesterol, and thereby stimulate the synthesis of LDL receptors. This formulation is supported by reports that lovastatin treatment induces messenger RNA for LDL receptors in liver in animals (2), and increases the fractional rate of removal of LDL from the circulation in humans $(3,4)$.

Very little information is available about the effects of HMG-CoA reductase inhibition on body cholesterol metabolism or homeostasis in intact humans. Sterol balance studies in five familial hypercholesterolemia heterozygotes showed that lovastatin caused a significant decrease $(22-44 \%)$ in the output of neutral and acidic steroids in three patients, but no alterations in two others (5). Similarly, lovastatin caused a moderate decrease in the urinary excretion of mevalonic acid $(6,7)$, a finding that was considered to reflect a moderate inhibition of body cholesterol synthesis.

Whole-body cholesterol turnover in humans can be studied by analyzing the turnover of plasma cholesterol after injection of radiolabeled cholesterol complexed with lipoproteins $(8,9)$. In previous work from this laboratory, we found that a three-pool compartmental model fitted the long-term cholesterol turnover curves in both normal subjects and in patients with a wide variety of abnormal serum lipid values (8-12). Analysis of turnover studies with this model provides estimates of the masses of exchangeable cholesterol and of the rates of flux of cholesterol in the pools and in the whole body. Four major model parameters, namely production rate (PR), the size of the rapidly exchanging compartment $\left(M_{1}\right)$, and the minimum values of the size of the slowly exchanging pool 3 $\left(\mathrm{M}_{3} \mathrm{~min}\right)$ and of total body exchangeable cholesterol $\left(\mathrm{M}_{\mathrm{tot}} \mathrm{min}\right)$, can be predicted by simple equations involving body weight, plasma lipid levels, and age (9). In contrast, neither the levels of HDL cholesterol or its apoproteins (11), nor those of HDL subfractions (12) provided additional predictive information about the parameters of whole body cholesterol metabolism.

We now report the results of a series of cholesterol turnover studies that were carried out in hypercholesterolemic patients before therapy, and then again at a later time while on longterm lovastatin therapy. The objective of the study was to determine whether reduction in plasma total and LDL cholesterol with lovastatin would be associated with a significant reduction in the whole-body cholesterol production (synthesis) rate, or in the sizes of exchangeable pools of body cholesterol. Lovastatin therapy to lower plasma cholesterol did not lead to

1. Abbreviations used in this paper: HMG, 3-hydroxy-3-methylglutaryl; PR, production rate. 
significant reductions in body cholesterol pools or in wholebody cholesterol synthesis.

\section{Methods}

Subjects studied. 12 volunteer subjects with high blood cholesterol levels were recruited for this study of the effects of drug treatment on total body cholesterol turnover. Written informed consent was obtained from each. The subjects were all patients, attending the Lipid Clinic of the Arteriosclerosis Research Center at the Columbia-Presbyterian Medical Center, who had previously undergone long-term cholesterol turnover studies (38-46 wk in duration). Before the initial (previous) turnover studies, the subjects had all been instructed in a diet containing reduced amounts of cholesterol $(<300 \mathrm{mg} / \mathrm{d})$ and of saturated fat $(<10 \%$ of calories), as described previously $(9,11)$. The subjects were all seen at regular intervals by a nutritionist, before and during the turnover studies, to ensure compliance with the diet. The results of these initial cholesterol turnover studies, conducted before the patients received drug therapy, have been reported previously $(9$, 11-13).

The clinical characteristics of the subjects are provided in Table I, where the patients are also identified by their previously assigned study numbers. At the time of the initial turnover studies a genetic classification of each subject was attempted, as described previously (9). Of the 12 subjects in the present study, five had been classified as familial hypercholesterolemia. In the other seven subjects, the presence or absence of a familial disorder was indeterminate (see Table 1).

The protocol for the study was as follows: $(a)$ recruitment of subjects who had previously had turnover studies; $(b)$ drug treatment for $15 \mathrm{mo}$, and $(c)$ repeat long-term cholesterol turnover study, while continuing on drug therapy. Nine subjects were treated with lovastatin, $20 \mathrm{mg}$ twice a day, to lower their total and LDL cholesterol levels. Treatment with lovastatin was begun at varying time intervals ( $1-5 \mathrm{yr})$ after the completion of the initial cholesterol turnover study. After 15 mo of lovastatin therapy, a new cholesterol turnover study was begun. The subjects continued lovastatin therapy, and remained at their drug-treatment steady-state lipid levels, throughout the duration of the study. The subjects in the lovastatin study were all on diets that were similar to those they had consumed at the time of the initial cholesterol turnover study. Specifically, the subjects consumed $<300 \mathrm{mg} / \mathrm{d}$ of cholesterol and $<10 \%$ of calories as saturated fat. As in the earlier turnover studies, all subjects were seen at regular intervals by a nutritionist, and compliance with the recommended diet was judged to be good. The observed characteristics of the subjects during the lovastatin

Table I. Characteristics of Subjects Studied

\begin{tabular}{|c|c|c|c|c|c|c|c|c|c|c|c|c|}
\hline Subject & $\begin{array}{c}\text { Drug } \\
\mathbf{R x}\end{array}$ & $\begin{array}{l}\text { No. in } \\
\text { previous } \\
\text { study* }\end{array}$ & Age & Sex & Height & Weight & IWT & CHOL & TG & LDL-C & HDL-C & $\begin{array}{l}\text { Familial } \\
\text { disorder }\end{array}$ \\
\hline
\end{tabular}

Lovastatin treated

\begin{tabular}{|c|c|c|c|c|c|c|c|c|c|c|c|c|}
\hline \multirow[t]{2}{*}{ A } & No & 60 & 58 & F & 159 & 59 & 113 & $320 \pm 20$ & $274 \pm 120$ & 206 & 55 & IND \\
\hline & Yes & & 66 & & 155 & 62 & 133 & $260 \pm 14$ & $273 \pm 93$ & 144 & 66 & \\
\hline \multirow[t]{2}{*}{ B } & No & 95 & 51 & $\mathbf{F}$ & 157 & 50 & 98 & $277 \pm 20$ & $233 \pm 88$ & 192 & 48 & FH \\
\hline & Yes & & 56 & & 157 & 52 & 102 & $257 \pm 15$ & $312 \pm 69$ & 154 & 47 & \\
\hline \multirow[t]{2}{*}{$\mathrm{C}$} & No & 55 & 52 & $\mathbf{M}$ & 183 & 87 & 119 & $276 \pm 16$ & $118 \pm 35$ & 193 & 54 & IND \\
\hline & Yes & & 55 & & 183 & 90 & 124 & $202 \pm 27$ & $92 \pm 11$ & 167 & 50 & \\
\hline \multirow[t]{2}{*}{ D } & No & 103 & 52 & $\mathbf{M}$ & 173 & 85 & 129 & $302 \pm 17$ & $293 \pm 84$ & 214 & 38 & FH \\
\hline & Yes & & 56 & & 173 & 89 & 134 & $232 \pm 32$ & $168 \pm 49$ & 161 & 49 & \\
\hline \multirow[t]{2}{*}{$\mathbf{E}$} & No & 105 & 46 & $\mathbf{M}$ & 183 & 100 & 137 & $279 \pm 14$ & $207 \pm 54$ & 195 & 45 & FH \\
\hline & Yes & & 50 & & 183 & 102 & 139 & $213 \pm 18$ & $155 \pm 22$ & 144 & 49 & \\
\hline \multirow[t]{2}{*}{$\mathbf{F}$} & No & 89 & 60 & F & 159 & 65 & 125 & $318 \pm 18$ & $211 \pm 107$ & 248 & 49 & IND \\
\hline & Yes & & 66 & & 159 & 69 & 132 & $244 \pm 22$ & $146 \pm 26$ & 181 & 50 & \\
\hline \multirow[t]{2}{*}{ G } & No & 111 & 64 & $\mathbf{M}$ & 188 & 74 & 101 & $346 \pm 28$ & $154 \pm 23$ & 261 & 42 & IND \\
\hline & Yes & & 67 & & 188 & 74 & 101 & $279 \pm 26$ & $128 \pm 40$ & 197 & 46 & \\
\hline \multirow[t]{2}{*}{$\mathbf{H}$} & No & 88 & 37 & $\mathbf{M}$ & 183 & 87 & 119 & $271 \pm 11$ & $170 \pm 50$ & 189 & 36 & IND \\
\hline & Yes & & 43 & & 183 & 98 & 134 & $229 \pm 25$ & $158 \pm 30$ & 146 & 39 & \\
\hline \multirow[t]{2}{*}{ I } & No & 100 & 27 & $\mathbf{M}$ & 183 & 88 & 111 & $259 \pm 27$ & $210 \pm 41$ & 188 & 46 & FH \\
\hline & Yes & & 32 & & 183 & 92 & 116 & $220 \pm 15$ & $171 \pm 33$ & 138 & 44 & \\
\hline \multicolumn{13}{|c|}{ Colestipol + niacin-treated } \\
\hline \multirow[t]{2}{*}{$\mathbf{J}$} & No & 78 & 55 & $\mathbf{M}$ & 162 & 70 & 119 & $251 \pm 28$ & $136 \pm 31$ & 207 & 43 & IND \\
\hline & Yes & & 59 & & 162 & 69 & 118 & $213 \pm 13$ & $214 \pm 32$ & 128 & 46 & \\
\hline \multirow[t]{2}{*}{$\mathbf{K}$} & No & J.R. & 34 & $\mathbf{M}$ & 173 & 64 & 100 & $277 \pm 18$ & $104 \pm 18$ & ND & ND & IND \\
\hline & Yes & & 41 & & 173 & 67 & 101 & $182 \pm 19$ & $108 \pm 21$ & 118 & 54 & \\
\hline \multirow[t]{2}{*}{ L } & No & 90 & 37 & $\mathbf{M}$ & 189 & 85 & 101 & $307 \pm 28$ & $96 \pm 14$ & 247 & 44 & FH \\
\hline & Yes & & 41 & & 189 & 84 & 107 & $207 \pm 18$ & $69 \pm 31$ & 148 & 60 & \\
\hline
\end{tabular}

Abbreviations: IWT, percent ideal body weight (see references 11 and 12); CHOL and TG, mean \pm SD values for total plasma cholesterol and triglyceride, respectively, during the period of study; LDL-C and HDL-C, mean LDL and HDL cholesterol levels during the period of study; ND, not determined; FH, familial hypercholesterolemia; IND, indeterminate. Age refers to age (in years) at the time the cholesterol turnover study was begun. * Subjects $25-54,55-84,85-111$ were first described in references 9,11 , and 12, respectively. Subject J.R. was first described in reference 13 . 
treatment study are shown in Table I. Subjects received no other lipidlowering drugs during the study, and the dosage of lovastatin was not altered. The subjects did not take any concurrent medication that might affect blood lipid concentrations. Before and during treatment with lovastatin, all patients had periodic ophthalmologic examinations and assessment of liver function blood enzymes and creatinine phosphokinase. No patient was discontinued from the study due to side effects of the drug.

Three patients who had previously had cholesterol turnover studies performed before receiving drug therapy had repeat cholesterol turnover studies carried out while being treated with the bile acid sequestrant resin colestipol hydrochloride $(20 \mathrm{~g} / \mathrm{d})$ plus niacin $(3-3.5 \mathrm{~g} / \mathrm{d})$. The patients had been receiving this combined drug therapy for 15-21 mo before the start of the repeat study, and continued on drug treatment throughout the turnover study. It is known that resin therapy increases the turnover and production rate of cholesterol (14). The characteristics of these subjects are also shown in Table I. These three subjects were studied at the same time as those receiving lovastatin, to allow for a comparison of the effects of the two different drug treatment regimens on the parameters of the three-pool model.

Cholesterol turnover studies and their analysis. $\left[4-{ }^{14} \mathrm{C}\right] \mathrm{Cholesterol}$ (New England Nuclear, Boston, MA), complexed with the subject's own serum lipoproteins, was injected intravenously, and the specific radioactivity of serum total cholesterol was determined in samples collected serially thereafter, as described in detail previously $(8,9,11)$. The amounts of radioactivity injected ( $\sim 25 \mu \mathrm{Ci}$ per subject) were measured precisely. Information about the turnover studies that were carried out before drug therapy has been reported in previous publications $(9,11-13)$. The repeat cholesterol turnover studies, carried out during drug therapy, were each $\sim 280 \mathrm{~d}$ in duration. 21 blood samples were obtained during each study, at times that were selected, based on the accumulated results of previous turnover studies, to provide good estimates of the parameters of the three-pool model. The total cholesterol and the specific radioactivity of cholesterol in each sample were determined as described previously $(8,9,11)$; these determinations were carried out in triplicate and the mean of the triplicate values used for each data point.

In each study, the specific radioactivity data were analyzed by a weighted, least squares technique (15) to determine the parameters of a three-pool mammillary model that would provide the best fit. The model and these analyses have been described in detail previously (8, 9). Six unique model parameters were determined: PR (cholesterol production rate in grams per day), $\mathbf{M}_{1}$ (size of pool 1 in grams), and the constants $k_{12}, k_{21}, k_{31}$, and $k_{13}$ (rate constants for transfer between pool 2 or 3 and pool 1 in days ${ }^{-1}$ ). Minimum values for the size of pool 2 $\left(M_{2}\right)$, of pool $3\left(M_{3}\right)$, and for the amount of total exchangeable body cholesterol $\left(M_{\text {tot }}\right)$ were computed by assuming that all new cholesterol enters pool 1.

Analytical and statistical procedures. In the initial turnover studies (before drug treatment), cholesterol and triglyceride concentration were measured using Autoanalyzer I methodology (Technicon Instruments Corp., Tarrytown, NY). In the repeat turnover studies (on drug therapy) these lipids were measured with automated enzymatic methods, (ABA 100, Abbott Laboratories, Chicago, IL). Cholesterol in LDL and HDL was measured using procedures specified for the Lipid Research Clinics Program (16) and the laboratory was standardized with the Centers for Disease Control.

Comparisons of lipid levels and model parameters before and during treatment were carried out using paired $t$ tests. Parameters for which predictive equations have been developed (9) were also analyzed by adjusting the observed difference in each parameter for changes in body weight and age and studying the significance of the adjusted differences by $t$ tests.

\section{Results}

Responses to cholesterol lowering therapy. As shown in Table I, lovastatin therapy reduced the levels of plasma total and
LDL cholesterol in every subject studied. The mean ( \pm SD) decrease in total cholesterol (see Table IV below) was $56.9 \pm 18.9 \mathrm{mg} / \mathrm{dl}$, representing a mean reduction of $19 \%$. The LDL cholesterol level was reduced by $50.5 \pm 13.3 \mathrm{mg} / \mathrm{dl}$ (mean \pm SD), a mean reduction of $24 \%$. These plasma cholesterol reductions were highly significant $(P<0.001)$. The plasma triglyceride level showed a mean decrease of $14 \%$ (mean \pm SD reduction $29.7 \pm 54.5 \mathrm{mg} / \mathrm{dl}$ ). HDL cholesterol levels showed no significant change with therapy (with a mean $\pm \mathrm{SD}$ change of $+2.9 \pm 5.4 \mathrm{mg} / \mathrm{dl}$ ).

The three patients treated with resin and niacin also showed substantial decreases in their plasma levels of total cholesterol (mean reduction 28\%) and LDL cholesterol (mean reduction $39 \%$ in the two subjects who had lipoprotein measurements; see Table I).

Effects of drug treatment on the model parameters of body cholesterol metabolism. Table II presents the model parameters calculated from each long-term cholesterol turnover study in each subject. The results are summarized, as mean $\pm S D$ values, in Table III. Table IV presents the changes seen with

Table II. Model Parameters of Cholesterol Metabolism for Each Subject

\begin{tabular}{cccccc}
\hline Subject & $\begin{array}{c}\text { Drug } \\
\mathbf{R x}\end{array}$ & $\mathrm{PR}$ & $\mathrm{M}_{1}$ & $\mathbf{M}_{3} \min$ & $\mathbf{M}_{\text {ToT }}$ min \\
\hline & & $g / d$ & $g$ & $g$ & $g$
\end{tabular}

Lovastatin-treated

$\begin{array}{llllll}\text { A } & \text { No } & 0.94 & 20.6 & 36.8 & 67.5 \\ \text { B } & \text { Yes } & 1.29 & 20.7 & 50.6 & 86.9 \\ & \text { No } & 0.81 & 14.4 & 20.5 & 50.1 \\ \text { C } & \text { Yes } & 0.95 & 19.3 & 32.1 & 73.4 \\ & \text { No } & 1.72 & 25.2 & 48.8 & 88.3 \\ \text { D } & \text { Yes } & 1.44 & 23.2 & 51.9 & 86.7 \\ & \text { No } & 1.33 & 21.5 & 59.2 & 108.4 \\ \text { E } & \text { Yes } & 1.48 & 26.9 & 111.8 & 175.7 \\ & \text { No } & 1.58 & 27.2 & 55.0 & 98.2 \\ \text { F } & \text { Yes } & 1.43 & 24.8 & 47.2 & 88.9 \\ & \text { No } & 0.69 & 17.9 & 47.7 & 70.1 \\ \text { G } & \text { Yes } & 0.73 & 15.9 & 43.1 & 65.1 \\ & \text { No } & 0.87 & 28.8 & 30.7 & 79.6 \\ \text { H } & \text { Yes } & 0.91 & 24.8 & 36.8 & 83.1 \\ & \text { No } & 1.12 & 30.1 & 36.6 & 88.9 \\ \text { I } & \text { Yes } & 1.18 & 27.0 & 38.2 & 92.7 \\ & \text { No } & 0.99 & 22.7 & 25.0 & 69.3 \\ & \text { Yes } & 1.15 & 23.1 & 30.1 & 81.0\end{array}$

Colestipol + niacin-treated

\begin{tabular}{llllll} 
J & No & 1.47 & 21.4 & 28.2 & 64.3 \\
& Yes & 2.69 & 29.9 & 36.6 & 92.3 \\
K & No & 1.00 & 23.9 & 26.9 & 70.7 \\
& Yes & 2.03 & 17.7 & 20.2 & 60.3 \\
L & No & 0.97 & 27.4 & 24.8 & 71.9 \\
& Yes & 2.54 & 25.6 & 21.6 & 68.1 \\
\hline
\end{tabular}

$M_{1}$ is the size of the rapidly turning pool of cholesterol; $M_{3} \min$ and $\mathbf{M}_{\text {тот }}$ min are, respectively, the minimal values of the size of the pools of slowly turning over and of total body exchangeable cholesterol. 
Table III. Mean ( \pm SD) Values for Physiological Variables and Model Parameters

\begin{tabular}{|c|c|c|c|c|}
\hline \multirow[b]{2}{*}{ Variable/parameter } & \multicolumn{2}{|c|}{ Lovastatin-treated $(n=9)$} & \multicolumn{2}{|c|}{$\begin{array}{l}\text { Colestipol + niacin-treated } \\
\qquad(n=3)\end{array}$} \\
\hline & No $R x$ & Yes Rx & No $R x$ & Yes Rx \\
\hline CHOL $(m g / d l)$ & $296 \pm 28$ & $237 \pm 25$ & $278 \pm 28$ & $202 \pm 15$ \\
\hline TG $(m g / d l)$ & $209 \pm 53$ & $178 \pm 70$ & $112 \pm 21$ & $130 \pm 75$ \\
\hline LDL-C $(m g / d l)$ & $210 \pm 27$ & $159 \pm 20$ & -* & - \\
\hline HDL-C $(m g / d l)$ & $46 \pm 7$ & $49 \pm 7$ & - & - \\
\hline $\operatorname{PR}(g / d)$ & $1.09 \pm 0.31$ & $1.17 \pm 0.27$ & $1.14 \pm 0.28$ & $2.42 \pm 0.34$ \\
\hline $\mathbf{M}_{1}(g)$ & $23.4 \pm 5.3$ & $22.9 \pm 3.7$ & $24.2 \pm 3.0$ & $24.4 \pm 6.2$ \\
\hline $\mathbf{M}_{3} \min (g)$ & $38.1 \pm 13.2$ & $49.0 \pm 25.7$ & $26.6 \pm 1.7$ & $26.1 \pm 9.1$ \\
\hline $\mathbf{M}_{\mathrm{TOT}} \min (g)$ & $78.6 \pm 50.1$ & $92.6 \pm 32.2$ & $69.0 \pm 4.1$ & $73.6 \pm 16.7$ \\
\hline
\end{tabular}

Abbreviations as in Table I.

* LDL-C and HDL-C (LDL-cholesterol and HDL-cholesterol) mean values are not given for the colestipol-treated patients, since lipoprotein data for the initial (before drug Rx) study were not available for all three patients.

drug therapy in each physiological variable and model parameter, and summarizes the results of the statistical analyses to estimate the significance of each of the observed changes. Fig. 1 shows representative data obtained from studies performed before and during lovastatin treatment ( $A$ : subject $\mathrm{I})$ and before and during resin-niacin therapy $(B$ : subject $\mathrm{L})$.

Two sets of statistical analyses were carried out with the results observed in the lovastatin-treated subjects (see Table

Table IV. Changes Seen with Drug Therapy

\begin{tabular}{|c|c|c|c|c|c|c|}
\hline \multirow{3}{*}{$\begin{array}{l}\text { Variable/ } \\
\text { parameter }\end{array}$} & \multicolumn{6}{|c|}{ Lovastatin treated } \\
\hline & \multicolumn{3}{|c|}{ All subjects $(n=9)$} & \multicolumn{3}{|c|}{ Omit subjects $B$ and $D(n=7)$} \\
\hline & Mean & SD & $P$ & Mean & SD & $P$ \\
\hline CHOL & -56.9 & 18.9 & $0.0001^{*}$ & -60.3 & 14.3 & $0.0001^{*}$ \\
\hline TG & -29.7 & 54.5 & 0.14 & -31.7 & 22.1 & $0.009^{*}$ \\
\hline HDL-C & +2.9 & 5.4 & 0.15 & +2.4 & 4.9 & 0.25 \\
\hline LDL-C & -50.5 & 13.3 & $0.0001^{*}$ & -52.0 & 14.3 & $0.0001^{*}$ \\
\hline PR & +0.08 & 0.14 & 0.13 & +0.06 & 0.2 & 0.34 \\
\hline $\mathbf{M}_{1}$ & -0.54 & 3.6 & 0.66 & -2.17 & 1.8 & $0.02^{*}$ \\
\hline $\mathbf{M}_{3} \min$ & +11.0 & 17.9 & 0.10 & +4.96 & 9.9 & 0.23 \\
\hline $\mathbf{M}_{\mathrm{tot}} \min$ & +13.9 & 22.6 & 0.10 & +4.99 & 10.0 & 0.23 \\
\hline
\end{tabular}

\begin{tabular}{lcrl}
\hline & \multicolumn{3}{c}{ Colestipol + Niacin-treated } \\
& \multicolumn{3}{c}{$(n=3)$} \\
\cline { 2 - 4 } & Mean & \multicolumn{1}{c}{ SD } & \multicolumn{1}{c}{$P$} \\
\hline CHOL & -76.6 & 33.8 & 0.06 \\
TG & +18.3 & 53.9 & 0.62 \\
PR & +1.27 & 0.3 & $0.015^{*}$ \\
M $_{1}$ & +0.17 & 7.5 & 0.97 \\
M $_{3}$ min & -0.50 & 7.9 & 0.92 \\
M $_{\text {tot }}$ min & +4.60 & 20.6 & 0.74 \\
\hline
\end{tabular}

Abbreviations as in Table I. The units of the physiological variables and model parameters are the same in Table III. The significance level ( $P$ value) was calculated by $t$ test.

* Values considered statistically significant.
A

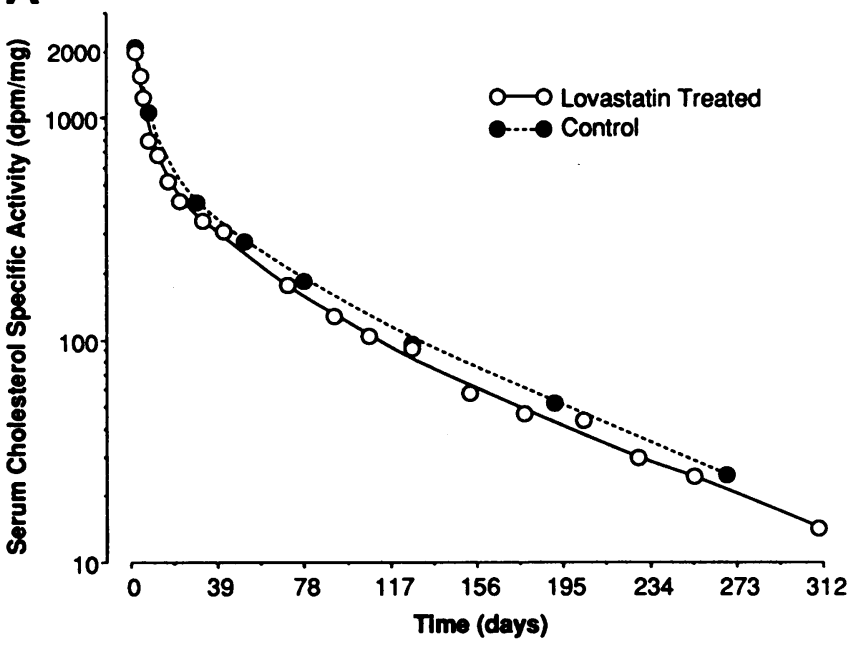

$\mathbf{B}$

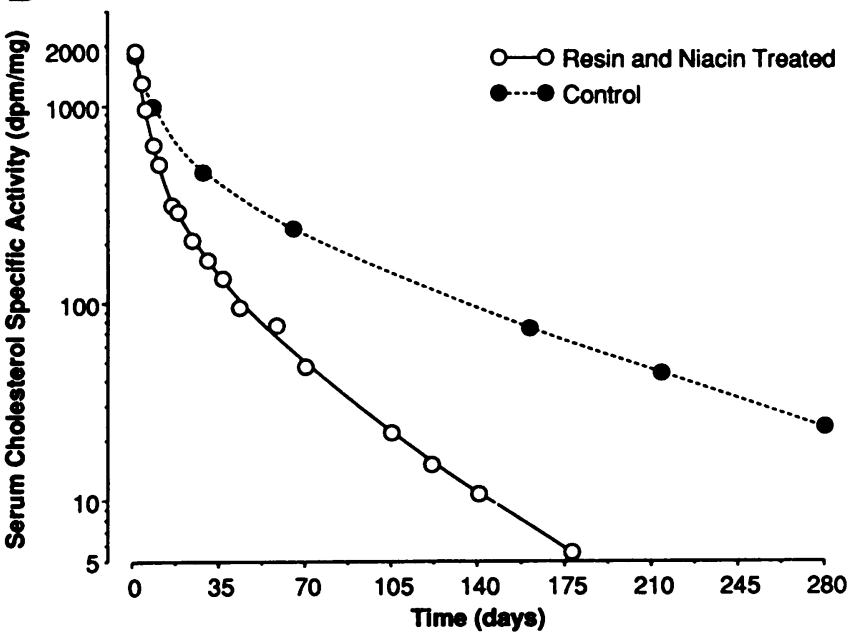

Figure 1. Turnover of plasma cholesterol before and during treatment with $(A)$ lovastatin or $(B)$ colestipol resin plus niacin. The studies shown are those that were carried out in subjects $\mathrm{I}(A)$ and $\mathrm{L}(B)$. The data points indicate the measured specific activity data for each study, normalized to an injected dose of $25 \mu \mathrm{Ci}$ of $\left[{ }^{14} \mathrm{C}\right]$ cholesterol. The curves are the computer-generated best fits of each set of data to the three-pool model.

IV). In the first set of analyses the data and results obtained from all nine subjects were analyzed. The analysis was then repeated omitting the results from subjects $\mathrm{B}$ and $\mathrm{D}$ (hence $n$ $=7$ in the second set of analyses). This was done because the studies in these two subjects were potentially flawed. Subject B had developed a rather considerable rise in triglyceride level during lovastatin treatment (from a mean of 233 to 312 $\mathrm{mg} / \mathrm{dl}$ ), which was considered unlikely to have been due to the effects of treatment. This change in circulating triglyceriderich lipoproteins, in part, reduced the effect of lovastatin to lower plasma cholesterol. For technical reasons, the analysis of the specific radioactivity data from subject $D$ appeared to be flawed, resulting in a small increase in $\mathbf{M}_{1}$ and an almost twofold apparent increase in $\mathbf{M}_{3}$ min on therapy. Thus, we wanted to determine whether elimination of the data from these two subjects with potentially flawed studies would alter the final conclusions in any way. 
Lovastatin treatment did not cause a significant change in the whole-body PR of cholesterol. Using the data from all nine subjects, the cholesterol PR was $1.09 \pm 0.31$ (mean \pm SD) $\mathrm{g} / \mathrm{d}$ before therapy, and $1.17 \pm 0.27 \mathrm{~g} / \mathrm{d}$ on drug therapy $(P=0.13$ by paired $t$ test). No change in PR was found, either, when subjects $B$ and D were omitted from the data analysis (see Table IV).

No significant changes associated with lovastatin therapy were noted in the sizes of the exchangeable pools of body cholesterol, when the complete data set ( $n=9$ subjects) was used for analysis (see Table IV). Thus, none of the changes, seen with drug therapy, in $\mathbf{M}_{1}$, in $\mathbf{M}_{3} \mathrm{~min}$, or in $\mathbf{M}_{\mathrm{tot}}$ min, were statistically significant. When subjects $B$ and $D$ were omitted from the data analysis ( $n=7$ subjects) the changes in $M_{3}$ min and $\mathbf{M}_{\text {tot }}$ min remained statistically not significant; however, with this data set $(n=7)$, a small but statistically significant $(P$ $=0.02$ ) decrease (mean of $2.2 \mathrm{~g}$ ) was seen in $M_{1}$, the size of the rapidly exchanging compartment.

In our earlier work (9) we showed (in a population of 54 subjects with a wide range of lipid levels) that $53-76 \%$ of the variation among humans in the four major model parameters studied here ( $P R, M_{1}, M_{3} \min$, and $M_{\text {tot }} \min$ ) could be explained by the variation in body weight, serum cholesterol, age, and adiposity. Using the predictive equations developed in this work (9), we could correct for the expected effects of changes in age and in body weight that occurred between the two studies in each subject. Accordingly, such an analysis was carried out to provide an examination of the effects of lovastatin therapy on the four model parameters corrected for the independent effects of changes in age and body weight. This analysis (data not shown) did not appreciably alter the sizes of the changes found in the model parameters as a result of therapy. No significant changes in the model parameters (on vs. before therapy) were found.

We also carried out analyses to search for possible correlations between the magnitude of the decrease in either total or LDL cholesterol level with lovastatin and any of the four model parameters or the change in model parameters seen with therapy. No significant correlations were observed ( $P$ $>0.05$ in every instance).

Treatment with colestipol resin plus niacin resulted in a marked, and over $100 \%$, increase in PR, from a mean value of 1.14 to $2.42 \mathrm{~g} / \mathrm{d}$ (see Tables III and IV). Only small, and statistically not significant, changes were seen in the sizes of the exchangeable pools of body cholesterol.

\section{Discussion}

These studies were conducted in order to explore the effects of lovastatin on the parameters of whole body cholesterol metabolism in intact human subjects. The results demonstrate that, whereas lovastatin therapy substantially reduced plasma total and LDL cholesterol levels, it did not significantly alter the total body cholesterol production rate, or the masses of exchangeable cholesterol in the rapidly exchanging compartment (pool 1), in the slowly exchanging compartment (pool 3), or in the total body.

With the three-pool model used here, cholesterol production rate is equivalent to the total body turnover rate (8). The PR is the steady-state rate at which new cholesterol molecules enter and leave the entire system (i.e., the body). Cholesterol molecules enter the body by de novo synthesis and by absorp- tion of cholesterol from the diet. Since the study subjects were all on similar, cholesterol-controlled diets $(<300 \mathrm{mg}$ intake per day) during both turnover studies, we can assume that cholesterol absorption was relatively small (of the order of 0.15 $\mathrm{g} / \mathrm{d}$ ) and of comparable magnitude in the two studies. Thus, the PR values reported here mainly represent cholesterol synthesis, and we can conclude that the total body synthesis rate of cholesterol was not significantly altered by lovastatin.

Our results extend and are somewhat at variance with those of Grundy and Bilheimer (5), who carried out sterol balance studies in five subjects and reported that lovastatin caused a moderate but significant decrease in total body synthesis of cholesterol in three subjects, and no change in two others. Evidence suggesting that treatment with HMG-CoA reductase inhibitors reduces body cholesterol synthesis has also been obtained in studies in which plasma or urinary concentrations of biosynthetic intermediates of cholesterol have been measured before and during therapy with lovastatin or simvastatin. Thus, both Parker et al. (6) and Pappu et al. (7) observed moderate decreases in the urinary excretion of mevalonate in patients receiving lovastatin. Kempen et al. (17) observed considerable decreases in the plasma levels of lathosterol in heterozygous familial hypercholesterolemia patients treated with simvastatin. Although measurements of these cholesterol intermediates have been shown to provide an index of the rate of body cholesterol synthesis as measured by sterol balance studies $(6,17)$, the measurements are indirect and their quantitative precision as a measure of body cholesterol synthesis is uncertain. Insull et al. (18) have also reported that lovastatin acutely inhibited whole-body cholesterol synthesis in four subjects, as measured by deuterium incorporation into erythrocyte free cholesterol.

In the sterol balance studies of Grundy and Bilheimer (5), patients received lovastatin for only rather short (up to $6 \mathrm{wk}$ ) study periods; different results might possibly have been observed after much longer periods of drug treatment. In the present studies, the subjects had been on lovastatin therapy, at new steady-state plasma lipid levels, for 15 mo before the repeat cholesterol turnover studies were begun. In addition, the fact that we did not observe a reduction in body cholesterol synthesis rate in the present study might be a chance result of patient selection. Thus, it is possible that on long-term lovastatin therapy some patients might show no change, whereas others show a mild to moderate reduction, in total body cholesterol synthesis. In our group of nine subjects, however, only two subjects (numbers $C$ and $E$, see Table II) showed PR reductions with lovastatin, and in both cases the extent of reduction was small. These results suggest that, in general, in patients on long-term treatment and in a new steady state, lovastatin has little or no effect on the total body synthesis rate of cholesterol.

This conclusion is somewhat surprising, since the mechanism of action of the drug is to interfere with cholesterol biosynthesis by inhibition of HMG-CoA reductase (19). Inhibition of this rate-limiting enzyme is thought to initially lower the cellular concentrations of cholesterol, leading to an increased expression of the genes that are subject to regulation by intracellular cholesterol. One of these genes is that of the enzyme (HMG-CoA reductase) itself (2). Thus, in vitro, marked increases in the levels of HMG-CoA reductase enzyme (20) and mRNA (21) have been seen after treatment with reductase inhibitors. Cells therefore respond to HMG-CoA reductase in- 
hibition by increasing the expression of the enzyme, thereby attempting to compensate for the inhibition of cholesterol synthesis and to increase cholesterol synthesis rate back towards the initial level.

The results reported here suggest that, in vivo in humans, the tissues of the body respond to the inhibition of HMG-CoA reductase produced by lovastatin by increasing the synthesis of the enzyme sufficiently to overcome the block in cholesterol synthesis rate. In the new steady state, on long-term lovastatin therapy, the tissues appear to fully compensate for the initial inhibition of cholesterol synthesis that must have been produced by the drug, leading to an unaltered total body cholesterol PR. An alternative explanation for the lack of effect of lovastatin on cholesterol PR is that lovastatin does produce a steady-state reduction in cholesterol synthesis in liver, but that this is offset by an increase in cholesterol synthesis in other tissues in response to the decreased levels of cholesterol in the circulation. We consider this a less likely explanation for reasons discussed below.

Studies on the molecular level have demonstrated the coordinate regulation of a number of genes by intracellular cholesterol. These genes include those for several enzymes in the pathway of cholesterol biosynthesis (HMG-CoA synthase [21], HMG-CoA reductase [21], prenyltransferase [22]), and the gene for the LDL receptor (2). These genes all share a common regulatory sterol response element in their $5^{\prime}$ upstream regions $(23,24)$. Two proteins have been identified by Gil et al. (25) that bind to the promoter region of the HMG-CoA reductase gene and may be important for regulation of gene expression. A zinc finger protein that binds to the sterol regulatory element has also recently been identified (26).

Inhibition of HMG-CoA reductase leads to a coordinate increase in the expression of all of these genes containing sterol regulatory elements. The effects on the LDL receptor are presumably responsible for the therapeutic effects of reductase inhibitors in hypercholesterolemic patients $(1,3,4)$. Increased LDL receptor activity in patients treated with lovastatin leads to an increased fractional rate of removal (clearance) of LDL from the circulation $(3,4)$, and hence to a reduced plasma level of LDL. Additional support for this mechanism (increased receptor-mediated clearance of $L D L$ ) is provided by the observation that lovastatin had no effect on LDL cholesterol levels in three children with homozygous familial hypercholesterolemia (27). These data suggest that the LDL receptor is required for lovastatin to exert a therapeutic effect. In addition, Malmendier et al. (28) studied LDL apoB turnover in seven normal subjects and concluded that the effect of simvastatin can be explained by an enhanced fractional removal of LDL from the circulation by the receptor route. Nevertheless, other mechanisms may also be operative in certain kinds of patients. For example, lipoprotein kinetic studies have shown that treatment of patients with combined hyperlipoproteinemia with lovastatin results in a decrease in the production rate of LDL with little change in LDL catabolism (29). Similarly, a decrease in LDL apo B production rate was reported to occur in a child with cholesteryl-ester storage disease who was treated with lovastatin (30). In some patients, lovastatin therapy may decrease triglyceride production rates (31). Thus, in some circumstances inhibition of HMG-CoA reductase may result in decreased LDL by either increasing LDL catabolism or decreasing lipoprotein production.

It is well established that treatment of hypercholesterol- emic patients with bile acid sequestrant resins leads to a marked increase in the rates of cholesterol synthesis and of receptor-mediated LDL clearance from the circulation, and to a consequent reduction in plasma total and LDL cholesterol levels (14). As expected, the three patients reported here who were studied while on resin plus niacin therapy showed marked increases in cholesterol PR. These patients were studied at the same time as, and for comparison with, the larger group of nine patients who were studied on lovastatin therapy. The results in the three resin plus niacin-treated patients illustrate the quantitative power of the kinetic method and model used here, and affirm the validity of the findings (of a lack of effect of treatment on the model parameters) in the lovastatin treated patients.

As discussed previously $(8,9)$, the finding that the longterm turnover of plasma cholesterol conforms to a three-pool model means that the various tissue pools of exchangeable body cholesterol fall into three groups in terms of the rates at which they equilibrate with plasma cholesterol. This concept has been validated by detailed tissue and whole-body studies in four baboons (32); it was found that the cholesterol turnover values for different tissues fell into three groups that were turning over at fast, intermediate, and slow rates, roughly comparable to the turnover rates calculated for the three pools of the model. Pool 1, which consists of cholesterol in fairly rapid equilibrium with plasma cholesterol, mainly consists of cholesterol in plasma, blood cells, liver, and intestines. Pool 2 consists of cholesterol which equilibrates at an intermediate rate with plasma cholesterol, and probably includes some of the cholesterol in viscera and in peripheral tissues. Adipose tissue cholesterol is an important part of the most slowly turning over compartment, pool 3. Cholesterol in other peripheral tissues, particularly connective tissue and skeletal muscle, and including arterial walls, also equilibrates slowly with plasma cholesterol and probably constitutes a significant portion of pool 3.

Lovastatin therapy did lead to a reduction in $M_{1}$, the size of the rapidly exchanging compartment. In the nine study patients, the mean value for $M_{1}$ before treatment was $23.4 \mathrm{~g}$. Plasma cholesterol would be expected to comprise $\sim 10.3 \mathrm{~g}$ in these patients, or $\sim 44 \%$ of $M_{1}$. The reduction in plasma cholesterol by lovastatin (mean 19\%) can hence be estimated to have reduced $M_{1}$ by a mean of $\sim 2.0 \mathrm{~g}$. If the cholesterol content of the liver and the other tissues that comprise pool 1 had been reduced to an extent similar to that seen in plasma, a mean decrease in $M_{1}$ of $4.4 \mathrm{~g}$ would have occurred. A decrease in $\mathbf{M}_{1}$ of this amount should have been detected easily by our methodology. A mean decrease of only $0.54 \mathrm{~g}$ (not significant, $P>0.6$ ) was, however, observed. Even when the results of two subjects with potentially flawed studies were omitted from the data analysis, the mean decrease in $M_{1}$ was only $2.1 \mathrm{~g}(P$ $=0.02$ ). These findings are consistent with the conclusion, discussed above, that the liver and other tissues compensate fully for the initial inhibition of cholesterol synthesis that must have been produced by lovastatin. Thus, in the new steadystate, cholesterol PR and tissue levels of cholesterol are not changed from the values seen before treatment, in association with an increased expression of the genes for both HMG-CoA reductase and the LDL receptor.

Cholesterol is an essential molecule, required for the normal structure of cell membranes and continued viability of mammalian cells. Accordingly, the cellular content and supply 
of cholesterol are homeostatically closely regulated (33). It could be argued that an agent which interferes with cholesterol biosynthesis and reduces tissue pools of cholesterol might be potentially toxic to cells in the long term. The results reported here, however, suggest that lovastatin at a therapeutic dose does not compromise tissue (and hence cellular) cholesterol content, and hence should not be worrisome in this respect with regard to long-term safety.

The lack of a reduction in the size of pool 3 might, at first glance, be disappointing. One might wish, for example, that an agent that reduces plasma cholesterol and is antiatherogenic would lead to a reduction in the size of the slowly exchanging and total exchangeable body pools of cholesterol. As discussed previously (11), however, cholesterol in atheromata represents a tiny fraction of the mass of pool 3 or of exchangeable body cholesterol. If there were effects of lovastatin that mainly influenced the mass of cholesterol in atherosclerotic plaque but had very little influence on the mass of other tissue pools of cholesterol, such effects would probably be undetected in the present studies. It is well established that the extent of atherosclerosis is largely determined by the plasma levels of total and LDL cholesterol $(34,35)$. Recent studies have shown, moreover, that aggressive cholesterol-lowering therapy can induce regression of atherosclerotic plaques in the coronary arteries of hyperlipidemic patients $(36,37)$. Thus, by markedly reducing the concentration of LDL in plasma, HMG-CoA reductase inhibitors apparently manifest desirable effects with regard to the prevention of atherosclerosis and coronary heart disease, without altering the major parameters of body cholesterol metabolism.

\section{Acknowledgments}

This work was supported by grant HL-21006 (SCOR) from the National Heart, Lung, and Blood Institute and by a grant from Merck, Sharp, \& Dohme, Inc. Dr. Goldberg is the recipient of an Established Fellowship from the American Heart Association, New York City Affiliate.

\section{References}

1. Grundy, S. M. 1988. HMG-CoA reductase inhibitors for treatment of hypercholesterolemia. N. Engl. J. Med. 319:24-33.

2. Ma, P. T., G. Gil, T. C. Sudhof, D. W. Bilheimer, J. L. Goldstein, and M. S. Brown. 1986. Mevinolin, an inhibitor of cholesterol synthesis, induces mRNA for low density lipoprotein receptor in livers of hamsters and rabbits. Proc. Natl. Acad. Sci. USA. 83:8370-8374.

3. Bilheimer, D. W., S. M. Grundy, M. S. Brown, and J. L. Goldstein. 1983. Mevinolin and colestipol stimulate receptor-mediated clearance of low density lipoprotein from plasma in familial hypercholesterolemia heterozygotes. Proc. Natl. Acad. Sci. USA. 80:4124-4128.

4. Grundy, S. M., and G. L. Vega. 1985. Influence of mevinolin on metabolism of low density lipoproteins in primary moderate hypercholesterolemia. J. Lipid Res. 26:1464-1475.

5. Grundy, S. M., and D. W. Bilheimer. 1984. Inhibition of 3-hydroxy-3-methylglutaryl-CoA reductase by mevinolin in familial hypercholesterolemia heterozygotes: effects on cholesterol balance. Proc. Natl. Acad. Sci. USA. 81:2538-2542.

6. Parker, T. S., D. J. McNamara, C. D. Brown, R. Kolb, E. H. Ahrens, Jr., A. W. Alberts, J. Tobert, J. Chen, and P. J. De-Schepper. 1984. Plasma mevalonate as a measure of cholesterol synthesis in man. J. Clin. Invest. 74:795-804.
7. Pappu, A. S., D. R. Illingworth, and S. Bacon. 1989. Reduction in plasma low density lipoprotein cholesterol and urinary mevalonic acid by lovastatin in patients with heterozygous familial hypercholesterolemia. Metab. Clin. Exp. 38:542-549.

8. Goodman, D. S., R. P. Noble, and R. B. Dell. 1973. Three-pool model of the long-term turnover of plasma cholesterol in man. J. Lipid Res. 14:178-188.

9. Goodman, D. S., F. R. Smith, A. H. Seplowitz, R. Ramakrishnan, and R. B. Dell. 1980. Prediction of the parameters of whole body cholesterol metabolism in humans. J. Lipid Res. 21:699-713.

10. Goodman, D. S., R. J. Deckelbaum, R. H. Palmer, R. B. Dell, R. Ramakrishnan, G. Delpre, Y. Beigel, and M. Cooper. 1983. Cholesterol turnover and metabolism in two patients with abetalipoproteinemia. J. Lipid Res. 24:1605-1611.

11. Blum, C. B., R. B. Dell, R. H. Palmer, R. Ramakrishnan, A. H. Seplowitz, and D. S. Goodman. 1985. Relationship of the parameters of body cholesterol metabolism with plasma levels of HDL cholesterol and the major HDL apoproteins. J. Lipid Res. 26:1079-1088.

12. Palmer, R. H., A. V. Nichols, R. B. Dell, R. Ramakrishnan, F. T. Lindgren, E. L. Gong, C. B. Blum, and D. S. Goodman. 1986. Lack of relationship in humans of the parameters of body cholesterol metabolism with plasma levels of subfractions of HDL or LDL, or with apoE isoform phenotype. J. Lipid Res. 27:637-644.

13. Smith, F. R., R. B. Dell, R. P. Noble, and D. S. Goodman. 1976. Parameters of the three-pool model of the turnover of plasma cholesterol in normal and hyperlipidemic humans. J. Clin. Invest. 57:137-148.

14. Goodman, D. S., R. P. Noble, and R. B. Dell. 1973. The effects of colestipol resin and of colestipol plus clofibrate on the turnover of plasma cholesterol in man. J. Clin. Invest. 52:2646-2655.

15. Dell, R. B., R. Sciacca, K. Lieberman, D. B. Case, and P. J. Cannon. 1973. A weighted least-squares technique for the analysis of kinetic data and its application to the study of renal ${ }^{133}$ xenon washout in dogs and man. Circ. Res. 21:71-84.

16. Lipid Research Clinics Program. 1974. Manual of Laboratory Operations: Lipid and Lipoprotein Analysis. National Institutes of Health Dept. of Health Education and Welfare Publication No. (NIH) 75-628. 1:74-81.

17. Kempen, H. J. M., J. F. C. Glatz, J. A. Gevers Leuven, H. A. van der Voort, and M. B. Katan. 1988. Serum lathosterol concentration is an indicator of whole-body cholesterol synthesis in humans. $J$. Lipid Res. 29:1149-1155.

18. Insull, W., D. L. Hachey, W. W. Wong, J. Jiang, B. W. Patterson, L. Clarke, and P. D. Klein. 1989. Acute effects of lovastatin on whole body cholesterol synthesis (WBCS) in man measured by deuterium incorporation. Arteriosclerosis. 9:741a. (Abstr.)

19. Alberts, A. W., J. Chen, G. Kuron, V. Hunt, J. Huff, C. Hoffman, J. Rothrock, M. Lopez, H. Joshua, E. Harris, et al. 1980. Mevinolin: a highly potent competitive inhibitor of hydroxymethylglutarylcoenzyme A reductase and a cholesterol-lowering agent. Proc. Natl. Acad. Sci. USA. 77:3957-3961.

20. Singer, I. I., S. Scott, D. M. Kazazis, and J. W. Huff. 1988. Lovastatin, an inhibitor of cholesterol synthesis, induces hydroxymethylglutaryl-coenzyme A reductase directly on membranes of expanded smooth endoplasmic reticulum in rat hepatocytes. Proc. Natl. Acad. Sci. USA. 85:5264-5268.

21. Mehrabian, M., K. A. Callaway, C. F. Clarke, R. D. Tanaka, M. Greenspan, A. J. Lusis, R. S. Sparkes, T. Mohandas, J. Edmond, A. M. Fogelman, et al. 1986. Regulation of rat liver 3-hydroxy-3-methylglutaryl coenzyme A synthetase and the chromosomal localization of the human gene. J. Biol. Chem. 261:16249-16255.

22. Rosser, D. S. E., M. N. Ashby, J. L. Ellis, and P. A. Edwards. 1989. Coordinate regulation of 3-hydroxy-3-methylglutaryl-coenzyme A reductase, and prenyltransferase synthesis but not degradation in HepG2 cells. J. Biol. Chem. 264:12653-12656.

23. Sudhof, T. C., D. W. Russell, M. S. Brown, and J. L. Goldstein. 1987. 42 bp element from LDL receptor confers end-product repres- 
sion by sterols when inserted into viral TK promoter. Cell. 48:10611069.

24. Osborne, T. F., J. L. Goldstein, and M. S. Brown. 1985. 5'end of HMG CoA reductase gene contains sequences responsible for cholesterol-mediated inhibition of transcription. Cell. 42:203-212.

25. Gil, G., T. F. Osborne, J. L. Goldstein, and M. S. Brown. 1988. Purification of a protein doublet that binds to six TGG-containing sequences in the promoter for hamster 3-hydroxy-3-methylglutarylcoenzyme A reductase. J. Biol. Chem. 263:19009-19019.

26. Rajavashisth, T. B., A. K. Taylor, A. Andalibi, K. L. Svenson, and A. J. Lusis. 1989. Identification of a zinc finger protein that binds to the sterol regulatory element. Science (Wash. DC). 245:640-643.

27. Uauy, R., G. L. Vega, S. M. Grundy, and D. W. Bilheimer. 1988. Lovastatin therapy in receptor-negative homozygous familial hypercholesterolemia: lack of effect on low-density lipoprotein concentrations or turnover. J. Pediatr. 113:387-392.

28. Malmendier, C. L., J.-F. Lontie, C. Delcroix, and T. Magot. 1989. Effect of simvastatin on receptor-dependent low density lipoprotein catabolism in normocholesterolemic human volunteers. Atherosclerosis. 80:101-109.

29. Arad, Y., R. Ramakrishnan, and H. N. Ginsberg. 1990. Lovastatin therapy reduces low density lipoprotein, apoB levels in subjects with combined hyperlipidemia by reducing the production of apoBcontaining lipoproteins: implications for the pathophysiology of apoB production. J. Lipid Res. 31:567-582.

30. Ginsberg, H. N., N. A. Le, M. P. Short, R. Ramakrishnan, and R. J. Desnick. 1987. Suppression of apolipoprotein B production during treatment of cholesteryl ester storage disease with lovastatin: im- plications for regulation of apolipoprotein B synthesis. J. Clin. Invest. 80:1692-1697.

31. Arad, Y., R. Ramakrishnan, and H. N. Ginsberg. 1989. Lovastatin therapy reduces the secretion of both VLDL apolipoprotein B and VLDL triglyceride in patients with combined hyperlipidemia. $A r$ teriosclerosis. 9:714a. (Abstr.)

32. Dell, R. B., G. E. Mott, E. M. Jackson, R. Ramakrishnan, K. D. Carey, H. C. McGill, Jr., and D. S. Goodman. 1985. Whole body and tissue cholesterol turnover in the baboon. J. Lipid Res. 26:327-337.

33. Brown, M. S., and J. L. Goldstein. 1986. A receptor-mediated pathway for cholesterol homeostasis. Science (Wash. DC). 232:34-47.

34. Reed, D. M., C. J. MacLean, and T. Hayashi. 1987. Predictors of atherosclerosis in the Honolulu Heart Program. I. Biologic, dietary, and lifestyle characteristics. Am. J. Epidemiol. 126:214-225.

35. Feinleib, M., W. B. Kannel, C. G. Tedeshi, T. K. Landau, and R. J. Garrison. 1979. The relation of antemortem characteristics to cardiovascular findings at necropsy: the Framingham Study. Atherosclerosis. 34:145-157.

36. Blankenhorn, D. H., S. A. Nessim, R. L. Johnson, M. Sanmarco, S. P. Azen, and L. Cashin-Hemphill. 1987. Beneficial effects of combined colestipol-niacin therapy on coronary atherosclerosis and coronary venous bypass grafts. JAMA (J. Am. Med. Assoc.) 257:32333240.

37. Brown, B. G., J. T. Lin, S. M. Schaefer, C. A. Kaplan, H. T. Dodge, and J. J. Albers. 1989. Niacin or lovastatin, combined with colestipol, regress coronary atherosclerosis and prevent clinical events in men with elevated apolipoprotein B. Circulation. 80(Suppl. II):266. 